\title{
Hemoptisis masiva en paciente con aspergilosis pulmonar: Hallazgos angiográficos y resolución endovascular
}

\author{
Massive hemoptysis in a patient with pulmonary aspergillosis
}

Ramiro Acevedo ${ }^{1}$, Raúl Solernó ${ }^{1}$, Agustín Ignacio Hauqui', Pablo Pedroni ${ }^{1}$, Ricardo Aquiles Sarmiento.

\section{RESUMEN}

La hemoptisis masiva es una entidad con etiologías variables, potencialmente mortal. Presentamos el caso de un paciente portador de aspergilosis pulmonar y hemoptisis masiva tratado por vía endovascular.

Palabras claves: hemoptisis, aspergilosis, tratamiento endovascular.

\section{ABSTRACT}

Severe haemoptysis may be a life threatening condition of variable aetiologies. In this article, a patient with a pulmonary aspergillosis and haemoptysis who required endovascular treatment is shown

Keywords: haemoptysis, aspergillosis, endovascular treatment.

Revista Argentina de Cardioangiología Intervencionista 2018;9(4):223-224. DOI: 10.30567/RACI/201804/0223-0224

\section{INTRODUCCIÓN}

La hemoptisis, definida como expectoración de sangre proveniente de las vías respiratorias bajas, es una emergencia médica potencialmente mortal. Cuando el sangrado supera los $300 \mathrm{ml}$ en 24 horas, se considera masiva ${ }^{9}$ y su elevada mortalidad bajo tratamiento conservador (50 a 100\% según diversas series) obliga a considerar una estrategia invasiva para su resolución. Es de destacar que la muerte se debe a hipoxia generada por la broncoaspiración de sangre y no por shock hipovolémico. Presenta diferentes etiologías: cáncer, vasculitis, infecciones pulmonares, embolismo pulmonar, malformaciones arteriovenosas (AV), biopsia pulmonar, etc. ${ }^{7}$ En la mayoría de los pacientes el sangrado se origina en las arterias bronquiales; sin embargo, otros vasos sistémicos no bronquiales también podrían ser fuente de sangrado ${ }^{4}$.

\section{CASO CLÍNICO}

Presentamos el caso de un paciente masculino de 61 años con antecedentes de diabetes, EPOC y aspergilosis pulmonar con mala adherencia al tratamiento, que consulta por progresión de disnea habitual hasta CF IV asociada a registros febriles. Se toma muestra de esputo que resulta positivo para Aspergillus niger, y se inicia tratamiento con anfotericina. Durante su evolución presenta hemoptisis asociada a hipoxemia sostenida requiriendo asistencia ventilatoria mecánica. Se realiza tomografía de tórax en la que se evidencia nódulo pulmonar con densidad de partes blandas en ápice pulmonar izquierdo en contacto con la pleura (Figura 1). La broncoscopia reveló sangrado activo en ambos bronquios lobares a predominio izquierdo y se procedió a instilar adrenalina con éxito tera-

1. Servicio de Cardiología Intervencionista. Hospital de alta Complejidad en Red - El Cruce

$\triangle$ Correspondencia: Dr. Ramiro Acevedo. Dirección: Av. Calchaquí 5401, Florencio Varela, CP 1888, Buenos Aires, Argentina. Teléfono: +541142109000 ext: 2794 E-mail: ramiroacevedo_86@hotmail.com

Los autores no declaran conflictos de intereses

Recibido: 21/06/2018|Aceptado: 27/08/2018 péutico inicial. Ante la recurrencia de hemoptisis se solicita angiografía, la cual se realiza por punción de arteria femoral derecha según técnica de Seldinger, colocando introductor 6 Fr. Se avanzó catéter pigtail sobre cuerda 0,035 ” hasta aorta torácica ascendente. Se realiza aortograma torácico mediante técnica de sustracción digital, constatándose la presencia de una fístula $\mathrm{AV}$ de arteria mamaria interna izquierda (AMI) hacia vena pulmonar (Figura 2), lo cual se confirma mediante inyecciones selectivas en dicha arteria con catéter IMA (Figura 3). Durante el estudio no se evidencia extravasación de contraste proveniente de las arterias bronquiales.

Se realiza intercambio de catéter por uno vertebral hidrofílico, posicionándolo en la arteria mamaria interna izquierda. Se procede a embolizar dicha arteria con un coil de $4,0 \mathrm{~mm}$ x $50 \mathrm{~mm}$ distal a la fístula mencionada y dos coils proximales de 5,0 $\mathrm{mm}$ x $60 \mathrm{~mm}$. Se constata correcta oclusión de AMI (Figura 4).

Evolución: el paciente evoluciona favorablemente, sin nuevo episodio de hemoptisis. Es dado de alta con tratamiento antifúngico oral.

\section{DISCUSIÓN}

La presencia de fístulas entre la AMI y los vasos pulmonares es una entidad poco común que puede tener diferentes etiologías: congénitas, vasculitis, trauma torácico, enfermedades infecciosas y la instrumentación de la arteria mamaria durante la cirugía de revascularización miocárdica ${ }^{1-3,5,6}$.

La fisiopatología de estas fístulas no es comprendida actualmente. Algunos autores postulan que la arteria mamaria interna puede verse involucrada en las lesiones del parénquima pulmonar y la pleura próximas a la pared anterior del tórax, como en nuestro caso ${ }^{4}$.

Las principales manifestaciones clínicas de esta entidad son accidente isquémico cerebral por embolia paradójica, disnea, angor, insuficiencia cardíaca y hemoptisis. Son con frecuencia halladas en forma incidental. Con respecto al examen físico, está descripta la auscultación de un soplo torácico continuo ${ }^{1,3}$.

El método para evaluar esta patología y su tratamiento aún no se encuentra protocolizado debido a la falta de guías clínicas hasta 


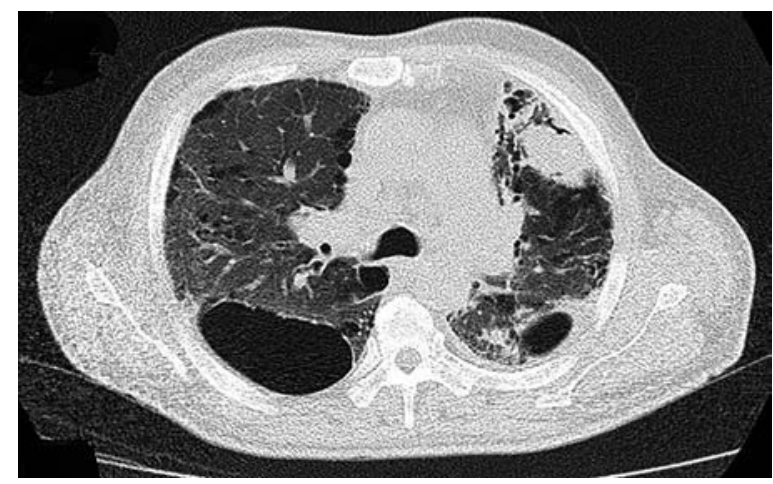

Figura 1. Imagen tomográfica de tórax. Nódulo pulmonar con densidad de partes blandas en ápice pulmonar izquierdo en contacto con la pleura.

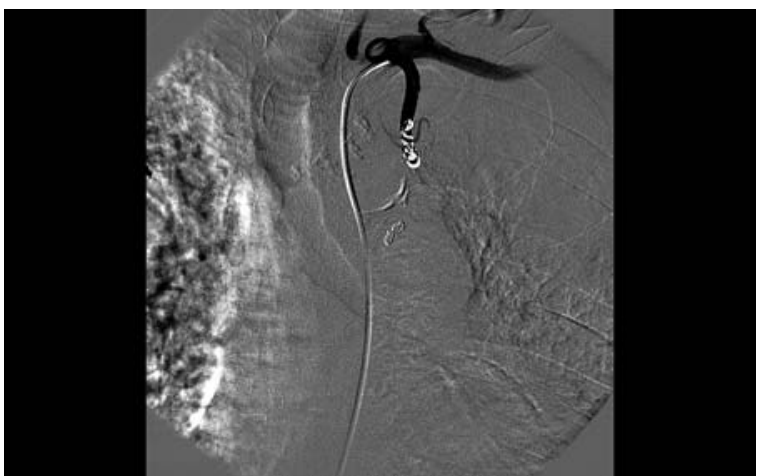

Figura 3. Angiografía selectiva de arteria mamaria izquierda. Lesión pulmonar hipervascularizada.

la fecha. El uso de una angiografía no selectiva (aortograma), facilita el reconocimiento y la disposición de las arterias bronquiales (anatomía muy variable), así como la presencia de arterias sistémicas no bronquiales, hipertrofiadas, que proveen vascularización transpleural a áreas de parénquima pulmonar anormal ${ }^{8}$. Existen numerosos agentes y dispositivos para obtener una adecuada oclusión vascular: agentes mecánicos permanentes (coils, plugs, oclusores vasculares y stents cubiertos), agentes particulados permanentes (partículas y esferas), agentes particulados temporarios (Spongostan) y agentes líquidos (esclerosantes, alcohol, cianoacrilato, Onyx $)^{1,3,4,6,8}$. En nuestro caso, utilizamos coils, los cuales fueron implantados en la AMI distal y proximalmente a la lesión, debido a la presencia de una fístula arteriovenosa con alto flujo hacia cavidades izquierdas. A pesar de no haberse detectado extravasación de contraste hacia el parénquima pulmonar, la presencia de ramas de la AMI tortuosas e hipertrofiadas que confluyen en una zona pulmonar anormal, o la constatación de pseudoaneurismas o fístulas AV, justifican su tratamiento endovascular ${ }^{3,4}$.

\section{BIBLIOGRAFÍA}

1. Jabbar AA, Patel A, Marzlin N, Altabaqchali S, Hasan M, Al-Zubaidi M, et al. Internal mammary artery-to-pulmonary vasculature fistula: Systematic review of case reports. Vascular Medicine 2017;22(5):426-31.

2. Ito T, Sakamoto T, Norio H, Kaji T, Okada Y. An arteriovenous Fistula between the Internal Mammary Artery and the Pulmonary Vein Following Blunt Chest Trauma. Cardiovasc Intervent Radiol 2005;28:120-3.

3. Jardin M, RemyJ. Control of Hemoptysis: Systemic Angiography and Anastomoses of the Internal Mammary Artery. Radiology 1988;168:377-83.

4. Hashimoto M, Heianna J, Okane K, Izumi J, Watarai J. Internal Mammary Artery Embolization for Hemoptysis. Acta Radiologica 1999;40:187-90.

5. Guler A, Yildiz M, Karabay CY, Aung SM, Aykan AC, KaragozA, GulerY, Esen

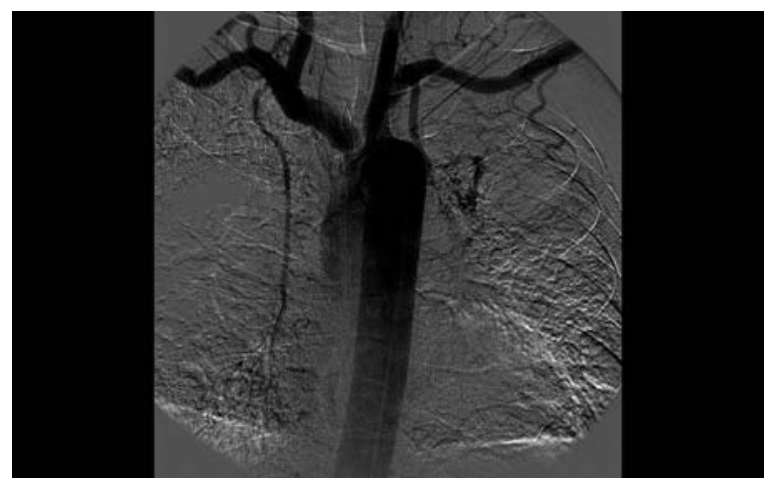

Figura 2. Aortograma torácico. Lesión pulmonar hipervascularizada.

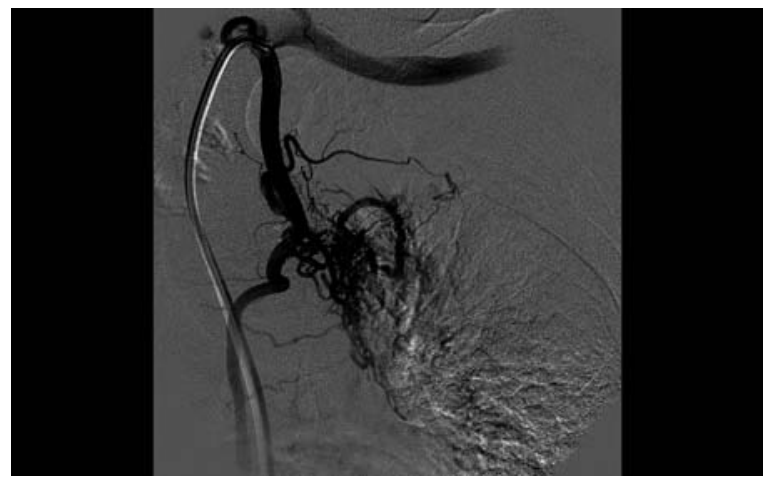

Figura 4. Correcta embolización de arteria mamaria interna izquierda

\section{CONCLUSIÓN}

La hemoptisis masiva, cualquiera sea su causa, es una entidad con alta mortalidad si no se toman medidas terapéuticas invasivas. En el caso de nuestro paciente, la etiología de la fístula entre la AMI y la vena pulmonar que desencadenó la hemoptisis fue la alteración en el parénquima pulmonar generada por un aspergiloma. La elección de coils como agente embolígeno se debió a la necesidad de obtener oclusión del vaso aferente en forma exclusiva, teniendo en cuenta que dicha lesión desembocaba en las cavidades cardíacas izquierdas y los vasos que la conformaban eran de gran calibre. Es de destacar que si bien la hemoptisis se debe a lesiones en las arterias bronquiales en el $90 \%$ de los casos, en el $5 \%$ de los casos el sangrado se origina en las arterias pulmonares y el $5 \%$ restante en arterias sistémicas no bronquiales ${ }^{7}$. Por lo anteriormente descripto, creímos importante realizar una angiografía panorámica como es el aortograma torácico, que fue lo que nos permitió identificar la malformación vascular causante del sangrado.
AM, Kirma C. Case series of a rare complication of CABG. Fistula between theinternal mammaryand pulmonaryvasculature. E-pub. Herz2013.DOI 10.1007/s00059-013-3814-2

6. Pierce G, Ahuja C, Chadha M. Case report: Complex internal mammary to pulmonary artery fistula as a cause of hemoptysis in tuberculosis: Diagnosis and endovascularmanagement usingethylenevinyl alcohol copolymer (Onyx). Indian Journal of Radiology and Imaging 2011;21(1):10-2.

7. Ittrich H, Bockhorn M, Klose H, Simon M. The Diagnosis and Treatment of Hemoptysis. Dtsch Arztebllnt 2017;114:371-81.

8. KesselD.Ray C. 2010. TranscatheterEmbolization and Therapy. Londres:Springer. 9. Kalva SP. Bronchial Artery Embolization. Tech Vasc IntervI Radiol 2009:12:130-8. 